Short communication

\title{
Improvement of bioelectrochemical property and energy recovery by acylhomoserine lactones (AHLs) in microbial electrolysis cells (MECs)
}

\author{
Wenzong Liu ${ }^{\text {a }}$, Weiwei Cai ${ }^{\mathrm{b}}$, Anzhou Ma ${ }^{\mathrm{a}}$, Ge Ren ${ }^{\mathrm{a}}$, Zhiling Li ${ }^{\mathrm{b}}$, Guoqiang Zhuang a , \\ Aijie Wang ${ }^{\text {a, b, * }}$ \\ a Key Laboratory of Environmental Biotechnology, Research Center for Eco-Environmental Sciences, Chinese Academy of Sciences, Beijing 100085, China \\ ${ }^{\mathrm{b}}$ State Key Laboratory of Urban Water Resource and Environment, Harbin Institute of Technology, Harbin 150001, China
}

\section{H I G H L I G H T S}

- Acylhomoserine lactones enhanced bioelectrochemcial activities in MECs.

- MEC performances were affected by chain length and concentration of AHLs.

- Highest $\mathrm{H}_{2}$ yield was achieved by 30C6-HSL at an initial concentration of $10 \mu \mathrm{M}$.

\section{A R T I C L E I N F O}

\section{Article history:}

Received 2 February 2015

Received in revised form

26 February 2015

Accepted 2 March 2015

Available online 3 March 2015

\section{Keywords:}

Bioelectrochemical system

Acylhomoserine lactone

Hydrogen yield

Quorum sensing
G R A P H I C A L A B S T R A C T

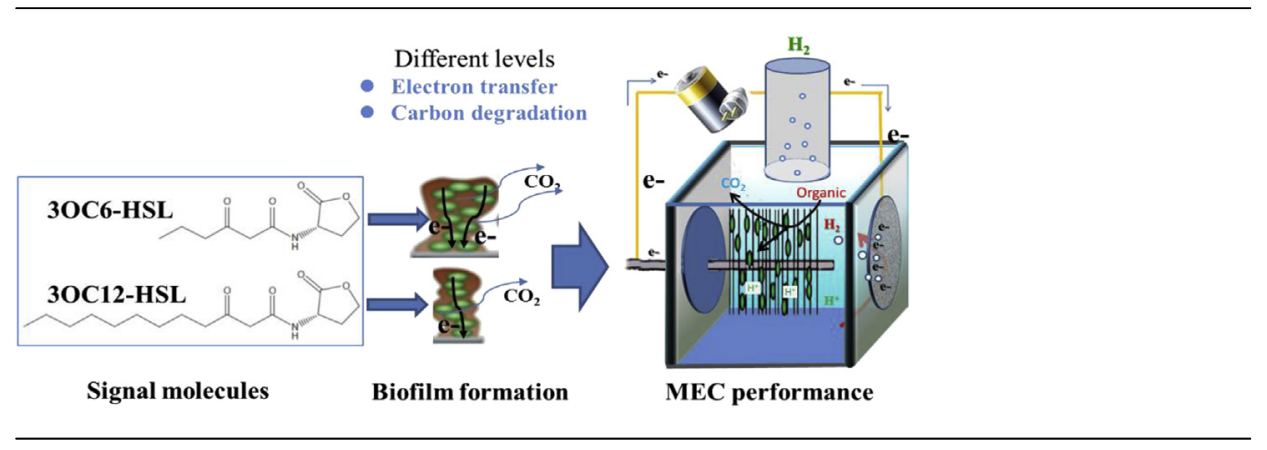

\section{A B S T R A C T}

Quorum sensing (QS) has been extensively studied as a cell-cell communication system, where small chemical signal molecules (acylhomoserine lactones, AHLs) can regulate the bacterial communications in bioelectrochemical systems via chemical signaling and electric signaling. In this study, electrochemical activity of bio-anode is substantially promoted by adding two kinds of AHLs with different chain length at the stage of community formation in microbial electrolysis cells (MECs). Hydrogen yield increase is observed by adding of two chain length AHLs, 3-oxo-hexanoyl-homoserine lactone (30C6-HSL) and 3oxo-dodecanoyl homoserine lactone (30C12-HSL). A higher MEC current is acquired with addition of 30C6-HSL than 30C12-HSL at a fixed voltage of $0.8 \mathrm{~V}$ (vs. SHE). The highest yield is up to $3.8 \pm 0.2 \mathrm{~mol}$ $\mathrm{H}_{2} \mathrm{~mol}^{-1}$ acetate at $10 \mu \mathrm{M}$ 30C6-HSL, which is increased 29\% over control MECs. Evaluated on applied voltage, energy efficiency is increased to $171.6 \pm 21.3 \%$ with short chain AHL, however, no significant improvement is performed on energy efficiency and coulombic efficiency with long-chain AHL. The study shows that bioelectrochemical characteristics of MECs varied on the chain length of AHL signal molecules and short-chain AHLs have a more positive effect on electron transfer and energy recovery in MECs.

() 2015 Elsevier B.V. All rights reserved.

\section{Introduction}

Quorum sensing (QS) signal molecules regulate the behavior of

\footnotetext{
* Corresponding author

E-mail address: waj0578@hit.edu.cn (A. Wang).
}

microbial organisms and directly facilitate microbial survival by promoting an advantageous lifestyle within a given environmental niche [1]. QS is a widespread phenomenon in microbial communities that employs autoinducing chemical signals to coordinate cooperative activities including biofilm formation [2,3] and extracellular enzyme secretion [4]. Recent works revealed that QS plays crucial roles in wastewater treatment on controlling the biological 
processes associated with biodegradation and bioelectrochemical activities [5]. The production of acylhomoserine lactones (AHL) or AHL-like molecules was observed in various biodegradation systems $[3,6]$. It was proved that significant amount of AHLs were produced by some bacteria like Pseudomonas sp. and thus QS regulation mechanisms were triggered to achieve a completely degradation on complex compounds [7]. Moreover, an enhanced electron transfer was manipulated by rhl AHL-mediated QS system, which has been verified in a pure culture accumulated microbial fuel cell (MFC) [8].

As a novel green energy technology, microbial electrolysis cells (MECs) have been proposed with great potential to degrade organic wastes and simultaneously recover energy for hydrogen generation as a sustainable energy carrier from waste with high efficiency [9-11]. The current studies has proved that MEC performed a higher energy recovery than MFC on over $90 \%$ coulombic efficiency and hydrogen yield from either simple organics or complex carbons [12]. Although various electron transfer mechanisms have been proposed [13-15], the efficiency of extracellular electron transfer is a major limiting factor that constrains the energy output in application of bioelectrochemical systems (BES). With the understanding that many anodic respiring bacteria related to proteobacteria can use AHLs as quorum-sensing signals [16], researches on QS activity have been directly carried out in MFCs, which have obtained positive effects on current generation by addition of signal molecules $[8,17]$. However, the signal effects vary in acyl group length and substitution depending on systems. Firstly, AHLs were much more significantly functioning on electron transport under anaerobic conditions without any oxidative stress [18]. However, potentially, hydrogen recovery in MEC requires a strict anaerobic/reductive condition, which was a different energy generation approach compared to MFCs under a microaerobic condition under air cathode [19]. Secondly, differences in acyl chain lengths are a factor in signal permeability. Short-chain acyl-HSLs, like N-3-oxo-hexanoyl-HSL (30C6-HSL) or butanoyl HSL, can diffuse freely through the cell membrane. While still diffusible, long-chain acyl-HSLs like $N$-3-oxo-dodecanoyl-HSL (30c12-HSL) appear to partition to the cell membrane [16]. Moreover, study showed that some bacteria were able to degrade long chain AHLs but not short chain AHLs as the sole carbon source [20].

The aim of the study was to investigate the energy recovery effects by addition of AHLs in MEC system. Two typical AHLs, shortchain acyl-HSL (3OC6-HSL) and long-chain acyl-HSL (3OC12-HSL), were taken to test effects on bioelectrochemical performance and energy recovery. The hydrogen yield and energy efficiency were discussed under different AHL concentrations at the beginning of MEC setup. To our knowledge, it is the first study to apply AHLs in MEC as a potential factor to improve the energy generation.

\section{Material and methods}

\subsection{MECs reactor setup and operation conditions}

Single chamber MEC reactors were set up with an effective volume was $38 \mathrm{~mL}$, including a $28 \mathrm{~mL}$ chamber $(3 \mathrm{~cm}$ diameter $\times 4 \mathrm{~cm}$ length) and a $10 \mathrm{~mL}$ gas collection tube as described in the previous study [21]. The anode was a graphite brush ( $3 \mathrm{~cm}$ diameter $\times 4 \mathrm{~cm} ; 0.22 \mathrm{~m}^{2}$ surface area) and while the cathode was made of carbon cloth (YW-50, YiBang; Taiwan) covered with a Pt catalyst layer ( $0.5 \mathrm{mg} \mathrm{Pt} \mathrm{cm}{ }^{-2}$ in one side) [22]. The reactors were operated in MEC model [19] and inoculated for two 24-h processes by mixture of PBS (50 mM; pH $=7.0$; $1500 \mathrm{mg} \mathrm{L}^{-1}$ acetate) and wastewater (V:V = 50\%:50\%) from local
Gaobeidian municipal WWTP in Beijing. Two typical AHLs of 30C6-HSL and 30C12-HSL were added in each inoculation process with two concentration levels of $1 \mu \mathrm{M}$ and $10 \mu \mathrm{M}$ respectively [2]. Triple reactors were applied in each condition and two for control without addition of any AHL. All reactors were applied with the same inoculum source of the wastewater for $24 \mathrm{~h}$. The external voltage was applied as $0.80 \mathrm{~V}$. The inoculation was repeated again with new addition of AHLs. After 48-h inoculation, all MEC reactors were refilled with PBS medium (50 mM; $\mathrm{pH}=7.0 ; 1500 \mathrm{mg} \mathrm{L}^{-1}$ acetate) every 24-h and maintained for at least 10 cycles to get stable hydrogen yield at room temperature $\left(25^{\circ} \mathrm{C}\right)$.

\subsection{Analysis and calculation methods}

Cyclic voltammetry was performed from -0.6 to $0.0 \mathrm{~V}$ (vs. SHE) with a scan rate of $2 \mathrm{mV} \mathrm{s}^{-1}$ using saturated calomel reference electrode (SCE, model-217, Shanghai Precise. Sci. Instru. Co., Ltd., China; $0.247 \mathrm{~V}$ vs. standard hydrogen electrode). The currents were automatic monitored (Acquisition system; Keithley Instrument) through a $10 \Omega$ resister. The gas was collected by a gas bag $(50 \mathrm{~mL}$; Cali5-Bond; Calibrated Instrument Inc) and gas components were analyzed by a gas chromatography (Fuli, GC9790; Zhengjiang instrument Inc, China) with a packed column [22] (TDX-01; $2 \mathrm{~m}$ length). The volume of generated gas was measured by a glass syringe. The VFAs were analysis by a gas chromatography (Agilent 4890; J\&W Scientific, USA) with a capillary column $(19095 \mathrm{~N}-123 \mathrm{HP}-\mathrm{INNOWAX} ; 30 \times 0.530 \mathrm{~mm} \times 1.00 \mathrm{ìm}$; J\&W Scientific, USA) [23].

The energy and coulombic efficiency were calculated to characterize the performance of MEC reactor [23]. Columbic efficiency indicated the recovery ability of electron, defined by the ratio of coulombs recovery to the total coulombs in substrate, which is integrated by current and time according to the equation $Q=I \times t$. Energy efficiency could be calculated on the $\eta=W_{\mathrm{H} 2} /$ $W_{E}=(n \times \Delta H) /(\mathrm{Q} \times$ Eap $)$ and the Eap was the external voltage; $n$ was the moles of hydrogen production in the standard conditions; $\Delta H$ was the combustion heat of $1 \mathrm{~mol}$ of hydrogen.

\section{Results and discussion}

\subsection{Enhanced bioelectrochemical properties of MECs by AHLs}

The ability of extracellular electron transfer is one limiting step from anodic communities to solid electrode, which fundamentally

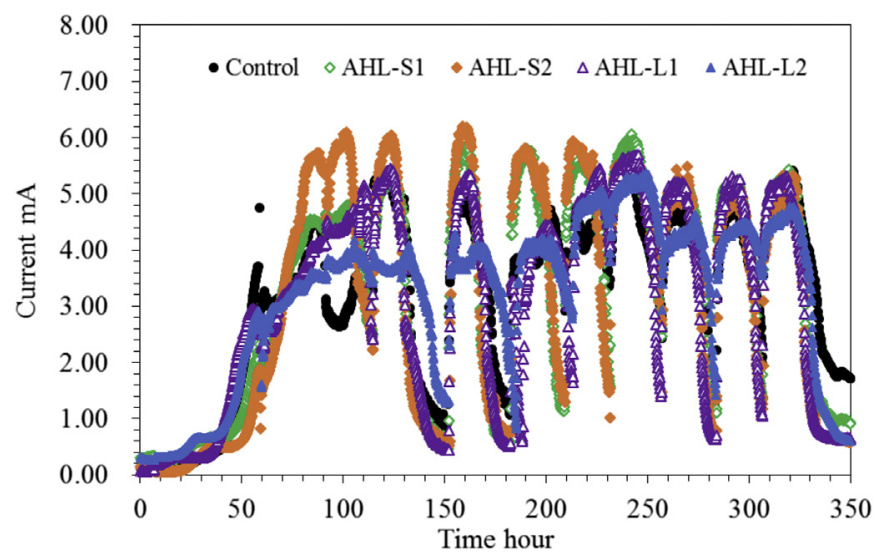

Fig. 1. Current generation of MECs after inoculation (two 24-h) with different addition of AHLs with an applied voltage of $0.8 \mathrm{~V}$ vs. SHE. 
determines the MEC efficiency. The positive effects were obtained on the initial current generation by addition of AHLs (Fig. 1). A quick electron transport was observed in MECs (applied voltage $0.8 \mathrm{~V}$ vs. SHE) with addition of short-chain 30C6-HSL after inoculation for 48-h. The highest peak current was achieved as $6.0 \mathrm{~mA}$ at the first initial cycles by addition of $10 \mu \mathrm{M}$ of 30C6-HSL, indicating a quick enrichment of anodic respiring bacteria. A later current increasing was shown in MECs with either $1 \mu \mathrm{M}$ of 30C6-HSL or $1 \mu \mathrm{M}$ of 30C12-HSL. Current was a little bit depressed with $10 \mu \mathrm{M}$ of longchain 30C12-HSL till $350 \mathrm{~h}$, although all other MECs performed similarly.

Cyclic voltammetry (CV) was used to present the redox activities and the stability of the species resulting from electron transfer in the anodic system of MECs. Exoelectrons were stored as accumulated charge in double layer capacitance formed between the biofilm and the electrode; this can be estimated by measuring the average between anodic current and cathodic current [24]. By comparison to control reactors without AHLs, addition of short and long chain AHLs enhanced a high capacity to accept electrons and charge accumulation during bioelectrolysis (Fig. 2). There was a noticeable increase on average current when a higher concentration of short chain AHLs (red line vs. pink line (in the web version) in Fig. 2). However, the long chain AHLs presented a quite similar effects on CV current under the two concentration levels (black and brown line). Generally, current with AHLs showed a much higher improvement than the blank (blue line). It seem that microbial communities, which were enriched with addition of the short chain AHLs with high concentration, presented the highest capacity to transfer electrons to anode.

The increased anodic peak current is resulted from a higher catalytic current, and more efficiently extracellular electrons transferred to the anode than the control. A much higher efficiency was achieved, which results in the enhancement of electrochemical activity in MECs. In this study, it was vital for anodic biofilm formation when AHLs were added only in inoculation process but not last over all operation time. Increased cell growth rate and generation of metabolites was also associated with improved substrate uptaking and/or metabolites excretion [8], because overexpression of key proteins is regulated by AHLs [25]. Under the regulation of AHLs in bioelctrochemical communities, bacteria can evolve to enhance the transport of endogenous electron shuttles across cell membrane to achieve more efficient extracellular electron transfer [26,27].

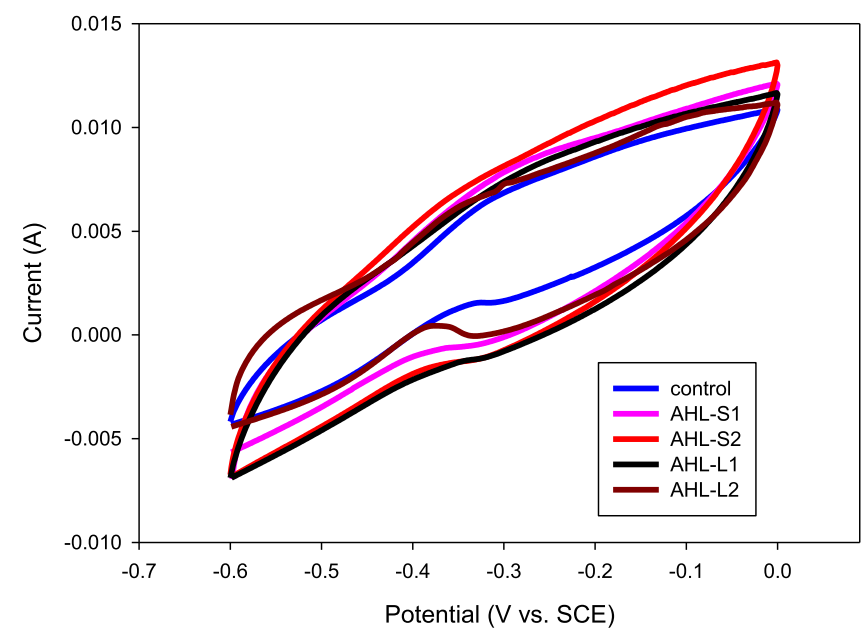

Fig. 2. Cyclic voltammograms of anodic biofilm under different addition of $1 \mu \mathrm{M} 30 \mathrm{C} 6$ HSL (AHL-S1), $10 \mu \mathrm{M}$ 30C6-HSL (AHL-S2), $1 \mu \mathrm{M}$ 30C12-HSL (AHL-L1), and $10 \mu \mathrm{M}$ 30C12-HSL (AHL-L2) in PBS medium at a scan rate of $2 \mathrm{mV} \mathrm{s}^{-1}$.

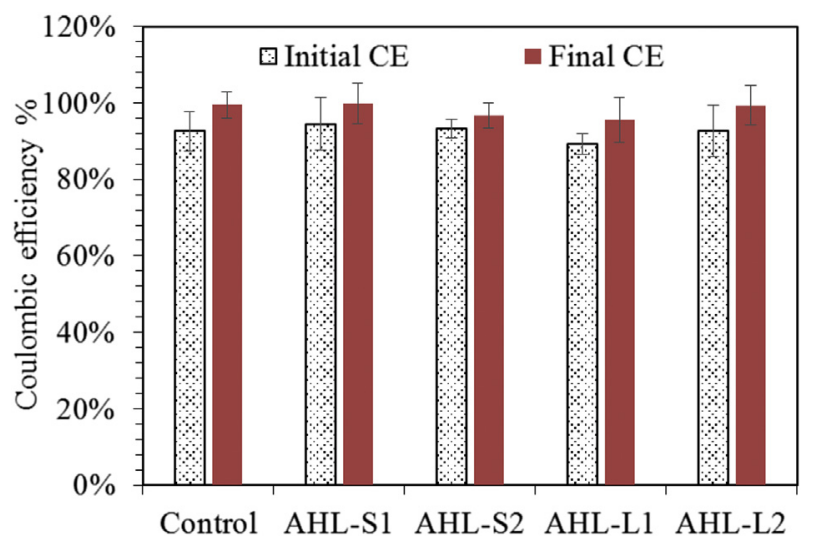

Fig. 3. Coulombic efficiency in different additions of AHLs.

\subsection{Energy recovery and hydrogen yield affected by additions of different AHLS}

The coulombic efficiencies (CE) varied in different AHL additions in the first four cycles after inoculation (Fig. 3). CE was $92.5 \pm 5.3 \%$ in control MECs. A little larger CE was achieved to $94.5 \pm 6.8 \%$ and $93.2 \pm 2.4 \%$ with $1 \mu \mathrm{M}$ and $10 \mu \mathrm{M}$ of 30C6-HSL, respectively. But no improvement was presented on CEs with long-chain 30C12-HSL. The positive effects from AHLs indicated a better electron transfer ability from acetate to anode in the MECs. At the end of the operation, there was no substantial difference of $\mathrm{CE}$ among all reactors and the averaged CE value was around 97.8\% (Fig. 3).

Hydrogen yield was calculated to evaluate bioelectrolysis system based on the acetate conversion. In comparison to control reactors, the hydrogen yields increased after the addition of AHLs (Fig. 4). The highest yield was up to $3.8 \pm 0.2 \mathrm{~mol} \mathrm{H}_{2} \mathrm{~mol}^{-1}$ acetate at $10 \mu \mathrm{M}$ 30C6-HSL, which was increased $29 \%$ over the control MECs. Accordingly, energy recovery efficiency was $171.6 \pm 21.3 \%$ with $10 \mu \mathrm{M}$ of 30C6-HSL. The hydrogen yield was $3.2 \pm 0.2 \mathrm{~mol}$ $\mathrm{H}_{2} \mathrm{~mol}^{-1}$ acetate with energy efficiency of $162.7 \pm 18.8 \%$ at low concentration of short-chain AHLs. There is no substantial improvements on energy recovery and coulombic efficiency by 30C12-HSL compared to the control reactor. The results indicated the short length of AHLs might play a quick effect on anodic biofilm communities [16].

It was proved that AHLs could facilitate the secretion of electron transfer mediators by some species like Pseudomonas aeruginosa [17], which was very supportable to explain the formation of anodic communities with few exoelectrogens and some Pseudomonas $[28,29]$. But the results indicated that short-chain AHLs play a prolonged effect to enhance the bioelectrochemical properties of

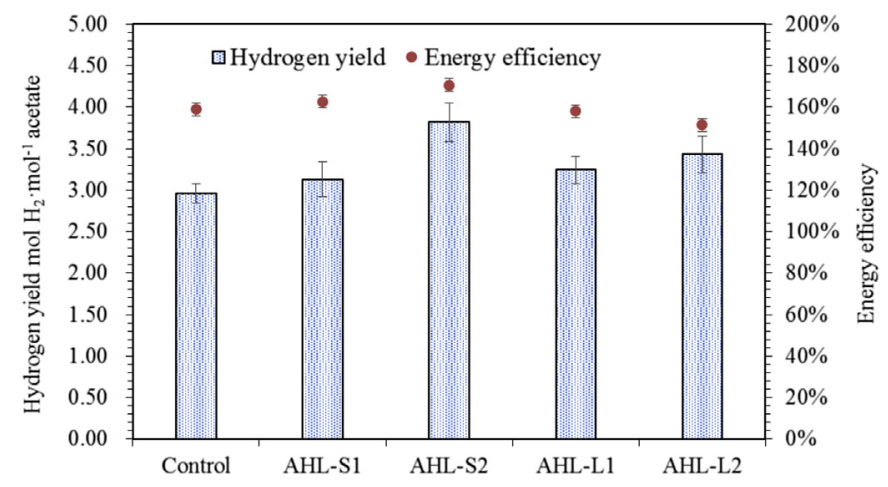

Fig. 4. Hydrogen yield and energy efficiency of MECs under different addition of AHLs. 
anodic communities. Besides the freely diffusion of shorter chain through the cell membrane, the possible reason is P. aeruginosa and Pseudomonas like species were able to degrade long chain AHLs but not short chain AHLs as the sole carbon source [20], though it was still not clear the correspondence between molar growth yields and AHL acyl side chain lengths. Degradation of the molecule prevents its sufficient accumulation in the anodic biofilm communities and will consequently lead to a disruption of the stimulated communication system [30]. It seem that AHLs addition likely reduced the start-up time of the electron transport (Fig. 1) and maintain the strengthened effects for a period without AHLs addition (with the residual) (Fig. 2). The enhancement was much more noticeable by short chain AHLs because long chain AHLs was hardly working in the culture supernatant fluid but almost all of the 30C6-HSL could spread quickly inside and outside the cells [31]. Therefore, the different effects were closely determined by the chain length throughout the study.

\section{Conclusion}

The study firstly revealed the addition of AHLs effectively improved hydrogen generation and energy recovery in MEC systems. The higher electron transfer activities were obtained with addition of short chain AHLs (30C6-HSL) than long-chain AHLs (30C12-HSL). The highest hydrogen yield was up to $3.8 \pm 0.2 \mathrm{~mol}$ $\mathrm{H}_{2} \mathrm{~mol}^{-1}$ acetate at $10 \mu \mathrm{M}$ of 30C6-HSL. The results showed that acylhomoserine lactones could regulate electric signaling in anodic biofilm formation to achieve an improvement on energy recovery in MECs.

\section{Acknowledgments}

This research was supported by National Science Foundation for Distinguished Young Scholars (Grant No. 51225802), National Natural Science Foundation of China (no. 51208496), National High-tech R\&D Program of China (863 Program, Grant No. 2012AA051502), Project 135 of Chinese Academy of Sciences (No. YSW2013B06), and 'Hundred Talents Program' of the Chinese Academy of Sciences.

\section{References}

[1] P. Williams, Microbiol.-SGM 153 (2007) 3923-3938.
[2] A. Valle, M.J. Bailey, A.S. Whiteley, M. Manefield, Environ. Microbiol. 6 (2004) $424-433$.

[3] T.T. Ren, X.Y. Li, H.Q. Yu, Bioresour. Technol. 129 (2013) 655-658.

[4] M. Schuster, D.J. Sexton, S.P. Diggle, E.P. Greenberg, Annu. Rev. Microbiol. 67 (2013) 43-63.

[5] Y.C. Yong, X.Y. Wu, J.Z. Sun, Y.X. Cao, H. Song, Chemosphere (2014), http:// dx.doi.org/10.1016/j.chemosphere.2014.10.020.

[6] G. Chong, O. Kimyon, S.A. Rice, S. Kjelleberg, M. Manefield, Microb. Biotechnol. 5 (2012) 621-633.

[7] Y.-C. Yong, J.-J. Zhong, Process Biochem. 45 (2010) 1944-1948.

[8] Y.C. Yong, Y.Y. Yu, Y. Yang, J. Liu, J.Y. Wang, H. Song, Biotech. Bioeng. 110 (2013) 408-416

[9] B.V. Ilje Pikaar, Stefano Freguia, Jurg Keller, Wastewater Treatment (Microbial Bioelectrochemical) and Production of Value-added By-products, Springer, New York, 2014.

[10] J.A. Turner, Science 305 (2004) 972-974.

[11] J. Gu, Y. Yan, J.W. Krizan, Q.D. Gibson, Z.M. Detweiler, R.J. Cava, A.B. Bocarsly, J. Am. Chem. Soc. 136 (2014) 830-833.

[12] S. Cheng, B.E. Logan, Proc. Natl. Acad. Sci. U. S. A. 104 (2007) 18871-18873.

[13] E. Marsili, D.B. Baron, I.D. Shikhare, D. Coursolle, J.A. Gralnick, D.R. Bond, Proc. Natl. Acad. Sci. U. S. A. 105 (2008) 3968-3973.

[14] G. Reguera, K.D. McCarthy, T. Mehta, J.S. Nicoll, M.T. Tuominen, D.R. Lovley, Nature 435 (2005) 1098-1101.

[15] L.A. Shi, D.J. Richardson, Z.M. Wang, S.N. Kerisit, K.M. Rosso, J.M. Zachara, J.K. Fredrickson, Environ. Microbiol. Rep. 1 (2009) 220-227.

[16] A.L. Schaefer, T.A. Taylor, J.T. Beatty, E.P. Greenberg, J. Bacteriol. 184 (2002) 6515-6521.

[17] Y.C. Yong, Y.Y. Yu, C.M. Li, J.J. Zhong, H. Song, Biosens. Bioelectron. 30 (2011) $87-92$.

[18] A. Venkataraman, M. Rosenbaum, J.B.A. Arends, R. Halitschke, L.T. Angenent, Electrochem. Commun. 12 (2010) 459-462.

[19] W. Liu, A. Wang, S. Cheng, B.E. Logan, H. Yu, Y. Deng, J.D. Nostrand, L. Wu, Z. He, J. Zhou, Environ. Sci. Technol. 44 (2010) 7729-7735.

[20] J.J. Huang, J.I. Han, L.H. Zhang, J.R. Leadbetter, Appl. Environ. Microbiol. 69 (2003) 5941-5949.

[21] S. Jiang, Y. Chen, Q. Zhou, Chem. Eng. J. 132 (2007) 311-317.

[22] W. Liu, S. Huang, A. Zhou, G. Zhou, N. Ren, A. Wang, G. Zhuang, Int. J. Hydrog. Energy 37 (2012) 13859-13864.

[23] A. Zhou, C. Yang, Z. Guo, Y. Hou, W. Liu, A. Wang, Biochem. Eng. J. 77 (2013) $240-245$.

[24] A. Cucu, T.A. Costache, M. Divona, A. Tiliakos, I. Stamatin, A. Ciocanea, Dig. J. Nanomater. Bios. 8 (2013) 1179-1190.

[25] W. Zhang, K. O'Connor, D.I.C. Wang, Z. Li, Appl. Environ. Microbiol. 75 (2009) 687-694.

[26] T. Zhang, C. Cui, S. Chen, X. Ai, H. Yang, P. Shen, Z. Peng, Chem. Commun. (2007), 3306-3306.

[27] Y. Qiao, C.M. Li, S.J. Bao, Z.S. Lu, Y.H. Hong, Chem. Commun. (2008) $1290-1292$.

[28] W.Z. Liu, A.J. Wang, N.Q. Ren, X.Y. Zhao, L.H. Liu, Z.G. Yu, D.J. Lee, Energ. Fuel 22 (2008) 159-163.

[29] W.Z. Liu, A.J. Wang, N.Q. Ren, Y.Q. Zhang, H.Y. Cheng, J. Biotechnol. 136 (2008) S635-S636.

[30] M.S. Medina-Martinez, M. Uyttendaele, A. Rajkovic, P. Nadal, J. Debevere, Appl. Environ. Microbiol. 73 (2007) 2329-2332.

[31] H.B. Kaplan, E.P. Greenberg, J. Bacteriol. 163 (1985) 1210-1214. 